arm (iv) Trial procedures - determine the appropriate primary outcome, adherence/compliance rates

Implications We aim to determine whether this approach is feasible and warrants further investigation in a larger randomised Phase II trial leading to a confirmatory multi-centre randomised Phase III trial to include a cost-effectiveness appraisal.

\section{P-102 USE OF OPIOIDS AS AN ADJUNCT IN THE MANAGEMENT OF SIALORRHEA IN MOTOR NEURONE DISEASE}

${ }^{1}$ Ee Jane Lim, ${ }^{2}$ Woon Fang NG, ${ }^{2}$ Ee Chin LOH, ${ }^{1}$ Matthew STAHL. 'University College London, UK; ${ }^{2}$ Universiti Malaya Medical Centre, Malaysia

\subsection{6/bmjspcare-2017-00133.101}

Background Current treatments for thin, watery hypersalivation in MND include conservative methods, anti-muscarinics, and Botulinum toxin injection or radiotherapy of the salivary glands (Young, Ellis, Johnson, Sathasivam, \& Pih, 2011).

Objective To report a case of sialorrhea in an MND patient in whom conventional treatment options were not helpful or tolerated. Morphine was trialled and found to reduce symptoms. Results W is a 42-year-old gentleman who was diagnosed with MND in 2013 needing BiPAP ventilation by January 2015. In February 2016, W presented with sialorrhea, and was started on $12.5 \mathrm{mg}$ Amitriptyline once nightly. However, he developed daytime somnolence, without significant symptom improvements and was switched to Benzhexol (Artane), $2 \mathrm{mg} /$ day in March. W was also trialled on Oramorph 2-4 mg PRN as an adjunct to Benzhexol, which he took about twice daily.

In August 2016, W presented with increased mucus plugging of his lower respiratory tract, intolerably dry oral mucosa, and thick oral secretions that became stuck at the back of his throat. Benzhexol was stopped and W underwent one session of radiotherapy. This only reduced his sialorrhea for 2 weeks, after which $\mathrm{W}$ developed salivary flooding every 1-2 hours. Morphine was then delivered via a continuous subcutaneous infusion (CSCI) $20 \mathrm{mg} / 24$ hours, which improved W's symptoms and alleviated salivary flooding.

As the MND progressed, W developed an ineffective cough and was unable to clear thick lower respiratory tract secretions. A cough assist machine was introduced.

W is currently on 25 mcg Fentanyl Patch (72 hourly) and a cough assist machine. W does not report any problems with daytime drowsiness, excessively dry oral mucosa, salivary flooding or thick secretions in the upper or lower respiratory tract.

Conclusion We propose that opioids have a measure of anticholinergic effects, which when used as an adjunct with existing therapies for MND, address the balance between excessive watery secretions and thick mucus plugging.

\section{P-103 A SYSTEMATIC REVIEW OF THE EFFECTIVENESS OF PALLIATIVE INTERVENTIONS TO TREAT RECTAL TENESMUS IN CANCER}

${ }^{1}$ Aine Ni Laoire, ${ }^{2}$ Lucy Fettes, ${ }^{2}$ Fliss E M Murtagh. 'Milford Care Centre, Ireland; ${ }^{2}$ Cicely Saunders Institute Kings College London, UK

10.1136/bmjspcare-2017-00133.102
Background Rectal tenesmus is a distressing symptom in patients with advanced cancer and challenging to treat. There is lack of consensus on the appropriate management of rectal tenesmus in this patient population.

Aim To identify and examine the effectiveness of interventions to palliate rectal tenesmus caused by advanced cancer when surgery, radiotherapy or chemotherapy are no longer treatment options.

Design A systematic review of the literature following standard systematic review methodology and the Preferred Reporting Items for Systematic Reviews and Meta-Analyses guidance.

Data sources A comprehensive search of the electronic databases MEDLINE, EMBASE and the Cochrane Library was conducted from the date of inception to April 2016. PubMed "related articles", grey literature, and hand-searches of the bibliographies of relevant papers and textbooks were also performed. Non-cancer patients were excluded. Any studies involving surgery or radiotherapy to treat tenesmus were excluded. Studies involving interventions to treat pelvic pain syndromes without specific outcome measures on severity of tenesmus were excluded. The quality of the studies was assessed using a National Institute for Health and Clinical Excellence recommended quality assessment tool.

Results From 861 studies, nine met full criteria and were selected. All were case series investigating the use of pharmacological interventions (diltiazem, nifedipine, methadone, mexiletine hydrochloride, lidocaine, bupivacaine), anaesthetic interventions (lumbar sympathectomy, neurolytic superior hypogastric plexus block), and endoscopic laser interventions. The included studies showed substantial heterogeneity and therefore a meta-analysis was not feasible.

Conclusion From this review we identified a significant gap in research into the palliation of rectal tenesmus. A multimodal approach may be necessary due to the complexity of the pathophysiology of tenesmus. Future research in this area should focus on randomised controlled trials of drug therapies whose potential effectiveness is suggested by case series'.

\section{P-104 RECOGNISING DYING IN ADULTS: IDENTIFYING PATIENTS IN THE LAST 12 MONTHS OF LIFE}

Zoe McKinstry. Salford Royal Foundation Trust, Manchester, Greater Manchester

\subsection{6/bmjspcare-2017-00133.103}

Background Recognising dying presents a challenge for healthcare workers, prognostic uncertainty can be a barrier to advanced care planning. NICE quality standard 13 Quality statement 1 states; People approaching the end of life are identified in a timely way. The GMC defines approaching end of life as when a person is likely to die within the next 12 months.

Method I conducted a retrospective audit of 27 patients who died in hospital over a 2 week period in 2016. Data was collected for 3 separate time intervals; 12 months- 6 months before death, 6 months to 7 days before death and the last 7 days of life.

Results At 12 months to 6 months before their death, most patients (19/27) met the criteria for Gold standard framework, however no patients had formal advanced care planning documented, 3/27 had DNA-CPR in place. At 6 months to 7 days before death all patients met criteria for GSF, more than could be identified as advanced or unstable disease, 3/27 had 
ACP documented. Most patients had hospital admissions during this time (23/27), more than one third of patients were referred to the Palliative Care Team. During the last 7 days of life 19/27 patients were recognised as dying, and had their wishes documented, all patients had DNA-CPR in place and 18/27 were referred to the Palliative Care Team.

Conclusions From this audit there were significant opportunities for healthcare workers to identify patients in the last 12 months of life, if this can be recognised sooner advanced care planning could be offered earlier to patients. These results suggest a need to make use of tools such as the Gold Standard Framework to better recognise patients who may be approaching end of life in order to help plan their preferred care.

\section{P-105 END OF LIFE CARE AT THE WEEKEND IN A UK DISTRICT GENERAL HOSPITAL}

Katharine Perry, Holly Randall, Katherine Webb. Royal Surrey County Hospital, Guildford, UK

\subsection{6/bmispcare-2017-00133.104}

Background In 2014 an independent review (Neuberger report) recommended discontinuation of the Liverpool Care Pathway. A subsequent report advised replacing the LCP with individualised end of life care plans, the contents of which were to be determined at local level.

The Supportive and Palliative Care Team (SPCT) at a UK District General Hospital and Cancer Centre developed a Personalised End of Life Care Plan (PELiCan) encompassing the five key priorities of care (One chance to get it right). Each patient on the PELiCan is assessed daily by a member of the SPCT. This study aimed to assess the use of the PELiCan and the utilisation of a seven day SPCT service.

Methods A database was created and every patient commenced on a PELiCan was entered. This database was retrospectively analysed over a 2 year period $\left(1^{\text {st }}\right.$ Sept 2014 and $31^{\text {st }}$ August 2016). Descriptive statistics were used to assess demographic details and diagnosis. The day each PELiCan started was noted as well as the date of death or discontinuation.

Results Nine-hundred and ten patients had an end of life care plan. The most common diagnosis was cancer (27\%), followed by diseases of respiratory system $(25 \%)$ and disease of circulatory system $(16 \%)$.

Twenty four percent $(n=222)$ of patients with a PELiCan died on a Saturday or Sunday. Five hundred and thirty-two patients $(58 \%)$ with a PELiCan were reviewed daily over the weekend. One-hundred and forty patients (15\%) were started with a PELiCan during the weekend (Saturday $n=56$, Sunday $\mathrm{n}=84$ ). Of these patients $34 \%$ had died before $9 \mathrm{am}$ on Monday morning.

Conclusions A significant proportion of end of life assessment and support occurs at a weekend. There is a requirement for seven-day palliative care services to implement effective specialist end of life symptom assessment.

\section{P-106 CHECKPOINT CHARLIE: AUDIT OF REGULAR SYRINGE DRIVER CHECKS ACROSS HEALTHCARE SETTINGS}

${ }^{1,2}$ Joseph Rassam, 1,2 Akash Chowdhury, 1,3,4 Karen Groves. 'West Lancs, Southport and Formby Palliative Care Services, Southport, UK; ${ }^{2}$ Liverpool University; Southport and Ormskirk Hospitals NHS Trust; ${ }^{4}$ Queenscourt Hospice, Southport

10.1136/bmjspcare-2017-00133.105

Background Syringe drivers are integral part to palliative care practice. The most widely used model, McKinley T34, is used to administer controlled drugs including opioids. As a result the documentation and monitoring of the use of syringe drivers is vital for ensuring safe treatment and in prevention or detection of potential adverse events.

Aim The aim of this audit was to review how effectively syringe driver checks and the subsequent documentation of such checks were carried out for patients in hospital, community and hospice settings.

Method Standards were set using current guidance and safety information. Patients were systematically identified through the help of the transform team (a palliative care liaison team), contacting the medical equipment library responsible for issuing the syringe drivers and by systematically going round the wards in the hospital setting. The checklists were analysed using a predetermined spreadsheet. A second improved checklist was introduced and the process repeated.

Results Data from the first cycle indicated the frequency of syringe driver checks was half that of the required 6 per day. A number of important safety parameters were under recorded and reasons identified included misleading questions and poor formatting of the syringe driver checklist (eg, questions on the reverse side of the page). After implementing the new sheet some modest improvements were made, most notably the documentation of syringe volume and syringe brand improved. Hospice and community data checklists were completed more accurately in accordance with clinical guidelines, compared to the hospital setting.

Conclusion The implementation of a checklist with clearer questions and an improved format resulted in some improvement. However the discrepancy between the hospital and community/hospice setting, where staff are more experienced with the use of syringe drivers, indicates the potential need for more training amongst hospital staff.

\section{P-107 IDENTIFICATION AND EVALUATION OF OBSERVATIONAL MEASURES FOR MONITORING SEDATION IN ADULT PALLIATIVE CARE PATIENTS: FINDINGS FROM A SYSTEMATIC REVIEW FOR I-CAN-CARE}

${ }^{1}$ Anna-Maria Krooupa, ${ }^{1}$ Bella Vivat, ${ }^{1}$ Bridget Candy, ${ }^{2}$ Stephen McKeever, ${ }^{1}$ Elena Marcus, ${ }^{1}$ Nuriye Kupeli, ${ }^{1}$ Paddy Stone. 'University College London, London, UK; ${ }^{2}$ Department of Children's Nursing, London South Bank University, London, UK

10.1136/bmjspcare-2017-00133.106 